\title{
Les belles idées de la défigurée : à propos du plateau labial des Mursi (Ethiopie)
}

The Beautiful Ideas of the Disfigured: About the Mursi Lip-Plate (Ethiopia)

Jean-Baptiste Eczet

\section{CpenEdition}

1 Journals

Édition électronique

URL : http://journals.openedition.org/imagesrevues/2501

DOI : 10.4000/imagesrevues.2501

ISSN : 1778-3801

Éditeur :

Centre d'Histoire et Théorie des Arts, Groupe d'Anthropologie Historique de l'Occident Médiéval, Laboratoire d'Anthropologie Sociale, UMR 8210 Anthropologie et Histoire des Mondes Antiques

Référence électronique

Jean-Baptiste Eczet, «Les belles idées de la défigurée : à propos du plateau labial des Mursi

(Ethiopie) », Images Re-vues [En ligne], 10 | 2012, mis en ligne le 23 octobre 2012, consulté le 04 mars 2021. URL : http://journals.openedition.org/imagesrevues/2501 ; DOI : https://doi.org/10.4000/ imagesrevues.2501

Ce document a été généré automatiquement le 4 mars 2021.

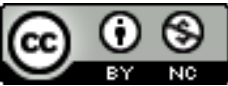

Images Re-vues est mise à disposition selon les termes de la Licence Creative Commons Attribution Pas d'Utilisation Commerciale 4.0 International. 


\section{Les belles idées de la défigurée : à propos du plateau labial des Mursi (Ethiopie)}

The Beautiful Ideas of the Disfigured: About the Mursi Lip-Plate (Ethiopia)

Jean-Baptiste Eczet

Le visage humain fut toujours mon grand

paysage.

Colette, Trait pour trait. 
1 Le plateau labial - ou labret - est pour le sens commun un des symboles de l'archaïsme. Comme les dites «femmes girafes » de Birmanie $^{2}$, on cite les "femmes à plateau» pour assoir le relativisme extrême de la beauté féminine. Souvent, celui-ci se conjugue avec, en arrière fond, la position moderne de témoin de pratiques ancestrales qui retourneront bientôt là d'où elles viennent. Loin de moi l'idée de vouloir répondre moralement à une fâcheuse tendance à l'évolutionnisme en positivant ce qu'est le labret. Par contre, il est intéressant de comprendre ce que les

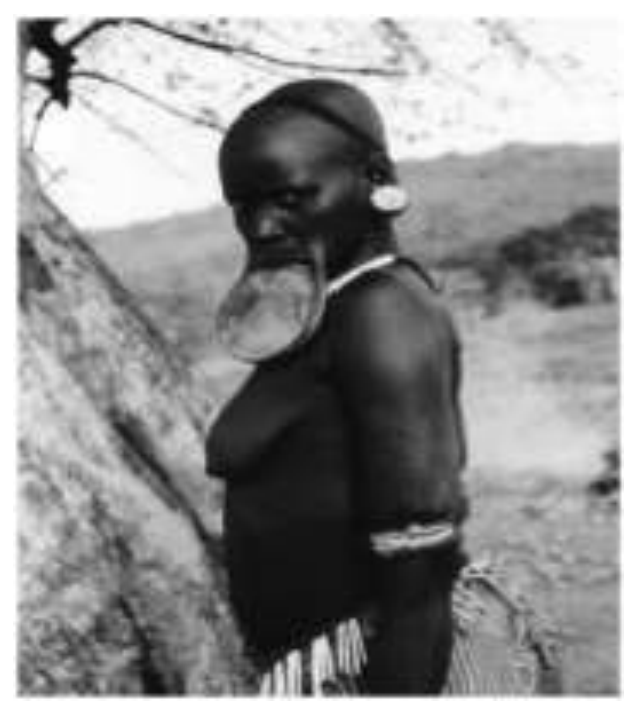
Mursi en disent, à savoir que « c'est bien » (a challi), que "ça fait mal» (a wadino) et qu'ils le font parce qu'ils sont Mursi. Le tout étant de ne pas produire de raisonnement identitaire circulaire en le décrivant comme un emblème mursi qui sert de signe distinctif, où la douleur ne s'inscrit que comme un supplément initiatique qui rend la proposition plus saillante. De même, considérer un unique facteur qui aurait pour conséquence de matérialiser le labret est peu probable. Un ornement corporel a plusieurs dimensions selon que le regard se porte sur la place qu'il a sur le corps et sa capacité à le modifier, sur sa matière, sa forme, son contexte d'usage ou sur la chaîne opératoire de sa fabrication. Or, le labret des Mursi est un ornement exceptionnel parce qu'il transforme un lieu précieux pour les interactions le visage -, parce qu'il produit une douleur lors de sa mise en place originelle et par la suite, parce qu'il gêne celles qui le portent et parce que sa forme n'a pas d'équivalent récurrent dans les artefacts des Mursi. Si nombres d'ornements corporels engendrent douleur, gêne ou modification de l'apparence - les Mursi en ont qui relèvent de chacune de ces dimensions -, il faut bien reconnaître que le labret les cumule avec une remarquable intensité. Il est de ces objets qui multiplient leurs façons d'être - et d'être vus - pour la simple raison que leur nature exceptionnelle n'est viable qu'à condition d'être le fruit d'un faisceau de représentations et d'actions qui convergent vers eux et les justifient. Le labret est donc un ornement objectivant un ensemble de choses encore à déterminer et s'il est un marqueur fort de l'identité mursi, c'est parce qu'il est au cœur d'un réseau d'idées et d'actions, loin de l'ornière identitaire discursive : « je suis Mursi donc je porte le labret car je suis Mursi » (fig. 1). 
Fig. 1

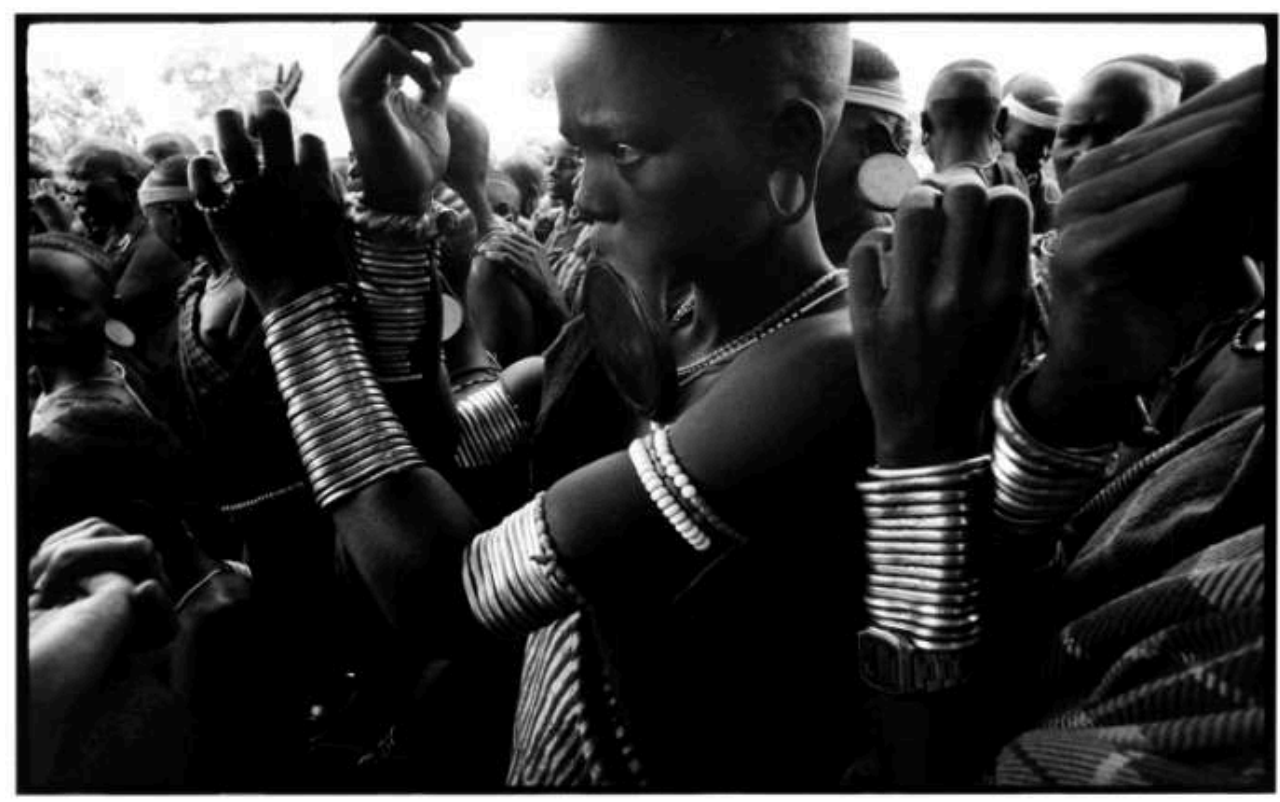

Jeune fille (dhole) portant un labret lors de danses. Maredunka, 2010. (photo : J.-B. Eczet)

\section{L'objet des femmes}

2 En raison de l'exotisme de cette incongruité ornementale, on court le risque de projeter notre ressenti dans les dispositions mursi jusqu'à relativiser le relativisme de la beauté en considérant que les Mursi aussi trouvent ça laid et le font à dessein. Que le lecteur m'excuse d'évoquer à nouveau le sens commun, d'autant plus au moyen d'une source collaborative où l'auteur s'est effacé derrière l'idée d'une vérité commune sans traçabilité, mais il me semble utile de débuter l'exposé en considérant le biais d'un fonctionnalisme trop stricte :

Certains anthropologues prétendent que cette mutilation labiale avait pour but de rendre inesthétiques les femmes afin de les protéger des razzias esclavagistes. De nos jours, la fonction serait uniquement symbolique puisque seules les femmes de caste élevée sont en droit de les porter. La taille du plateau est à la mesure de la dot exigée par la famille des jeunes filles à marier, dot composée de bovins et caprins, etc. ... d'une arme à feu, modernité et insécurité obligent ${ }^{3}$ !

3 Outre quelques erreurs ${ }^{4}$, le plateau ne se pense qu'en termes de fonction explicite, qu'on le voit avec dégout («rendre inesthétique les femmes») ou avec des yeux culturalistes ("à la mesure de la dot exigée »). Pour le dire autrement, un tel ornement ne peut exister que si une volonté consciente et absolument réflexive l'a voulue: quelque chose de si laid doit servir une cause ${ }^{5}$. Il apparait pourtant que le labret est pour les Mursi une forme qui s'impose, tant elle est évidente, et que son action sur le monde peut se passer d'un contenu discursif et d'une justification réflexive. Les Mursi n'ont pas besoin d'en parler pour faire entrer le labret dans leurs agissements quotidiens car cette forme épuise son sens sans même recourir aux commentaires.

4 Le labret est un disque d'argile, parfois de bois. On le désigne principalement par la matière dont il est constitué : dhebbi (pl. dhebbinia) pour ceux en argile, kio (pl. keno) pour ceux en bois. Les premiers sont d'abord une boule de terre argileuse humidifiée et 
longuement frappée afin d'en extraire les poches d'air et d'augmenter sa densité. Petit à petit, cette boule est aplatie et un disque apparait. Une fois que celui-ci atteint le diamètre voulu, les doigts mouillés de la femme qui le fabrique dessinent sur la tranche un sillon large d'une lèvre, le plus lisse et régulier possible. Il est ensuite jeté dans les braises d'un feu et, selon le procédé de cuisson et d'oxydation, deviendra noir, rouge, brun ou blanc ${ }^{6}$. Le labret de bois (aussi appelé burgui), plus rare, demande plus de temps car aucun outil spécifique n'existe pour le faire émerger d'un tronc. On doit régulièrement recouvrir un morceau de bois avec de la graisse qui, en l'imprégnant, le rend tendre et facilite sa découpe. Il faut noter que les disques placés dans les lobes d'oreilles sont identiques bien que de plus petit diamètre. Les labrets cassent vite et nécessitent d'en fabriquer souvent. En tant qu'objet, ils ne sont pas sujets à une valorisation particulière. C'est leur intégration au corps qui produit un résultat particulier. Qu'ils soient désignés par leur matière seulement n'est pas qu'une métonymie mais plutôt la reconnaissance qu'un labret sans corps, sans femme, sans lèvre, n'est qu'un bout d'argile ou un bout de bois.

La lèvre inférieure, comme beaucoup de bouts de peau visibles et non tendus, se prête bien au perçage et à l'ornementation. Les femmes Me'en qui vivent au nord du territoire des Mursi se percent aussi la lèvre au moyen d'un bâtonnet de bois, mais, à la différence des Mursi, elles n'augmentent pas la taille du trou par la suite. Seul un cône en argile, dont la pointe est orientée vers la gencive et la base arrondie vers l'extérieur, est maintenu. Les Nyangatom qui vivent au sud des Mursi ont, hommes comme femmes, un ornement dans la lèvre inférieure, tantôt bande métallique au bout de laquelle pend parfois une perle, tantôt grosse perle seule maintenue par une courte tige de bois.

Contrairement aux femmes Me'en et Nyangatom qui portent leur labret de manière continue, les femmes Mursi le portent dans des contextes où l'on peut juger la personne sur des critères perceptibles visuellement sans forcément la connaître en propre. Aussi, les contextes cérémoniels et cérémonieux sont-ils propices au port du labret, c'est-àdire durant les danses et autres moments de séduction, lorsqu'une épouse sert un repas à son mari et ses hôtes, et lors de la plupart des rituels qui sont, pour les Mursi, les seuls moments réunissant plus de personnes que le voisinage immédiat. Les moments où le labret s'impose sont assez bien définis et, dans tous les cas, l'absence des hommes rime avec l'absence du labret (fig. 2). 
Fig. 2

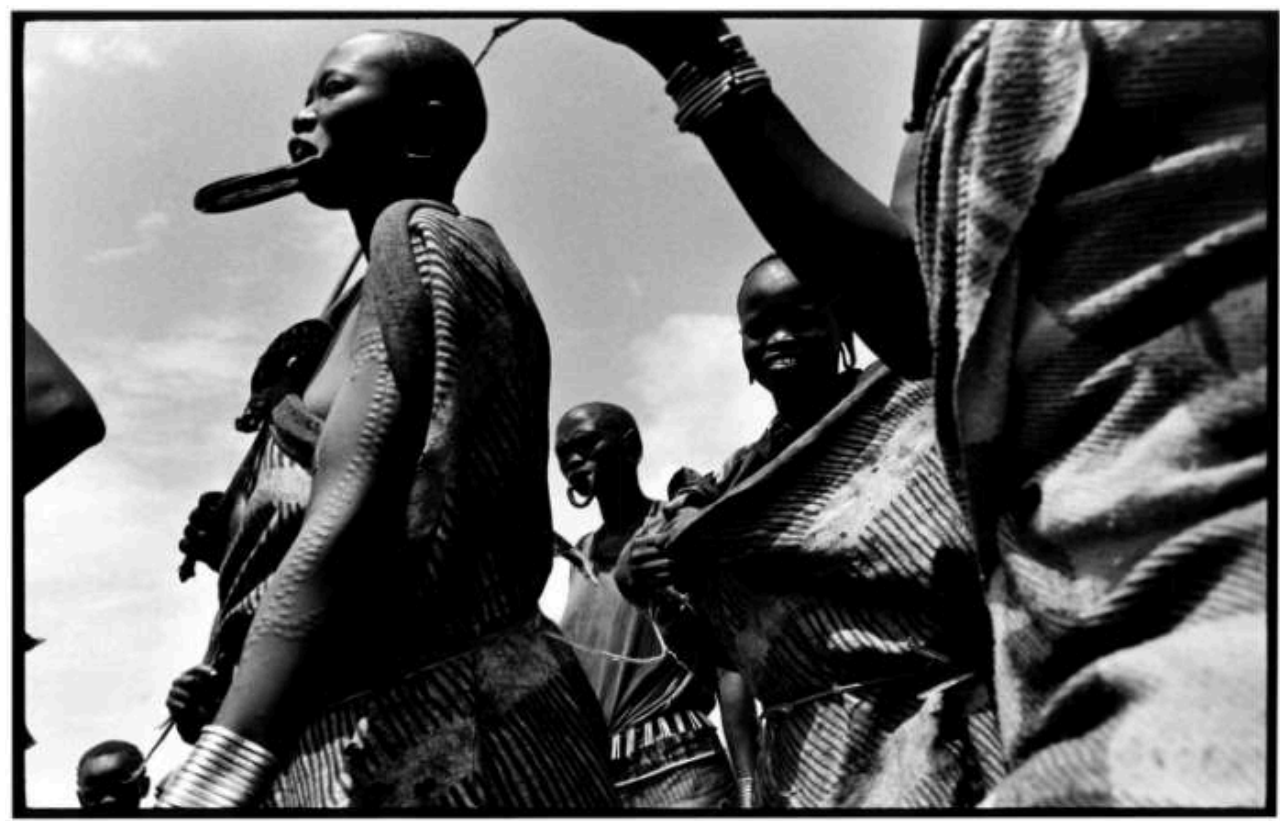

Groupe de femmes lors du passage de l'une d'elles de « jeune fille » (dhole) à « épouse » (moe). Miso, 2010 (photo : J.-B. Eczet)

\section{De la femme-objet}

7 Il en est du plateau comme des talons hauts : il n'est pas nécessaire de mobiliser trop longuement l'affordance ${ }^{7}$ pour convaincre que le port d'un labret ne va pas de soi et qu'il ne permet pas de faire ce que l'on veut. Son « invite » n'est pas évidente et sa mise en place des plus délicate. Le schème postural qui prend en compte cet objet s'adapte, gestes et tenue se modifient : le port de tête est altier, la démarche souple et rasante, et le face-à-face devient la modalité interactionnelle privilégiée, sans quoi le plateau se balancerait dangereusement, viendrait taper le sternum jusqu'à, cela arrive, déchirer la lèvre. Conséquence de la restriction motrice: on ne peut porter le plateau que dans des activités précises et des moments relativement délimités - et l'exercice prend tout son sens, le porter dans les activités domestiques (traite, préparation de la farine, soins aux enfants, etc.) relève de la haute voltige : essayez donc de passer l'aspirateur en talons hauts. La comparaison dépasse l'anecdote. Shauna LaTosky relève aussi la modification de la gestuelle qui est recherchée :

Bi Kalomi was teasing me about getting my lip cut and mimicking the way in which a girl should walk while wearing it: her head held high, her chin swaying back and forth in a very subtle, sensual manner ${ }^{8}$.

Le labret se détecte avant tout dans les manières d'être. Si détecter les intentions d'autrui selon les actions qu'on le voit faire est le propre de la théorie de l'esprit, alors le labret est un guide pour orienter les inférences. Le corps, dans les rails du labret, se meut autour d'un bout d'argile ou de bois qui rend plus prégnantes la gravité, l'inertie, la force centrifuge. Il s'adapte et adopte les gestes les plus efficaces dans le cadre de cette contrainte. Le spectateur infère alors de ce corps mouvant une impression de solennité, qui n'est autre que le retournement de la contrainte en avantage : le corps « empêché » montre aux spectateurs l'attention qu'il leur porte (fig. 3). 
Fig. 3

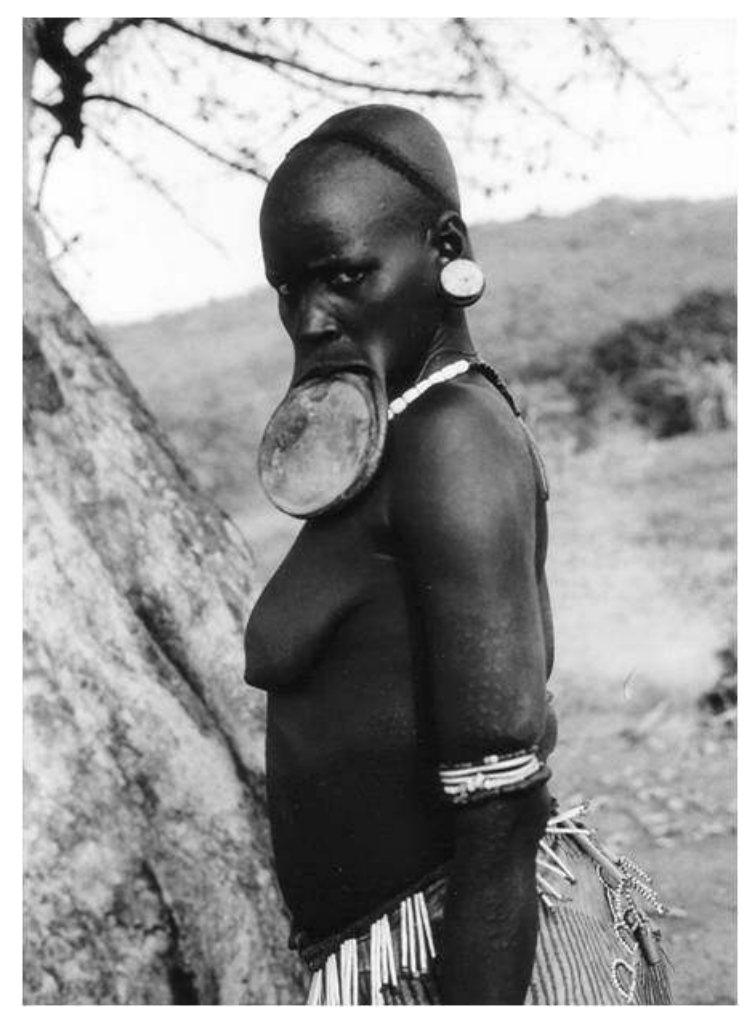

Le port du labret a un impact sur l'ensemble du schème corporel. Mako, 2008 (photo : J.-B. Eczet)

LaTosky parle de conscience de soi (self-consciousness) pour décrire la conscience aigüe de la femme pour la gestuelle valorisée par les hommes. Elle cite aussi un de ses informateurs, Oli Lori :

If she didn't put it in I would hit her and mother would hit her. Later, when she is given to her husband, if she does not put in her lip-plate, her husband will hit her. We teach her how to behave for when she goes to her husband?

10 Mais la femme n'exprime pas qu'une déférence. Car, au-delà de l'acceptation de la contrainte, elle doit bien se comporter et peut même faire mieux. Le message est double avec celui, d'une part, de faire avec la contrainte, et celui, d'autre part, de s'en servir: acceptation et dépassement. On préfère les petits pas aux grands, le peu de parole aux longs discours criards, les gestes mesurés à l'emphase corporelle et les femmes prennent aussi l'initiative du labret pour produire l'effet escompté ${ }^{10}$. Bref, la méta proposition que donne la femme qui porte son labret - et qui n'est pas donnée par l'objet lui-même - est celle de l'intériorisation du point de vue de l'homme sur la femme au moyen d'une gestuelle spécifique, dans des contextes de séduction ou de présentation valorisée de soi qui ne font pas appel à des éléments intimes.

11 Si le plateau était une forme univoque de domination masculine (seulement de l'acception sans dépassement), il y a bien longtemps que ces bouts d'argiles resteraient en terre car la violence familiale et conjugale tient ce rôle très bien, comme le précise Oli Lori ci-dessus. À intérioriser le point de vue des hommes concernant certaines de leurs attentes à propos des femmes, ces dernières montrent à ces mêmes hommes qu'elles y arrivent, avec tout le bénéfice qu'elles peuvent en tirer. De même, en véhiculant le point de vue des hommes sous forme de gestuelle corporelle, elles en 
rendent témoin les autres femmes et les plus jeunes. On est en fait une personne bien pour tout le monde, les autres femmes comprises.

\section{À la femme-sujet}

12 Mais le labret n'est pas tout le temps porté. Quand il est déposé, outre une gestuelle désormais modifiée car partiellement intégrée même sans labret, un trou béant reste sur le visage. Et cette absence dessine en creux le point de vue des femmes sur ellesmêmes.

13 La lèvre inférieure pend par-delà le menton. Quelques muscles donnent à cette partie de la lèvre une certaine mobilité mais elle reste le plus souvent inerte. Plusieurs gestes s'adaptent à cette marque qui invalide une partie de la bouche. La langue mun ne possède pas de dento-labiales fricatives comme le $\mathrm{f}$ ou le $\mathrm{v}$, et évite les occlusives bilabiales en attaque des mots, principalement le p. Par exemple, les occidentaux sont génériquement appelé farenj' ou farenji par les populations des hautes terres, auquel les Mursi substituent le terme arenji. De même, la police est dite oliso, dérivé de poliso (fig. 4).

Fig. 4

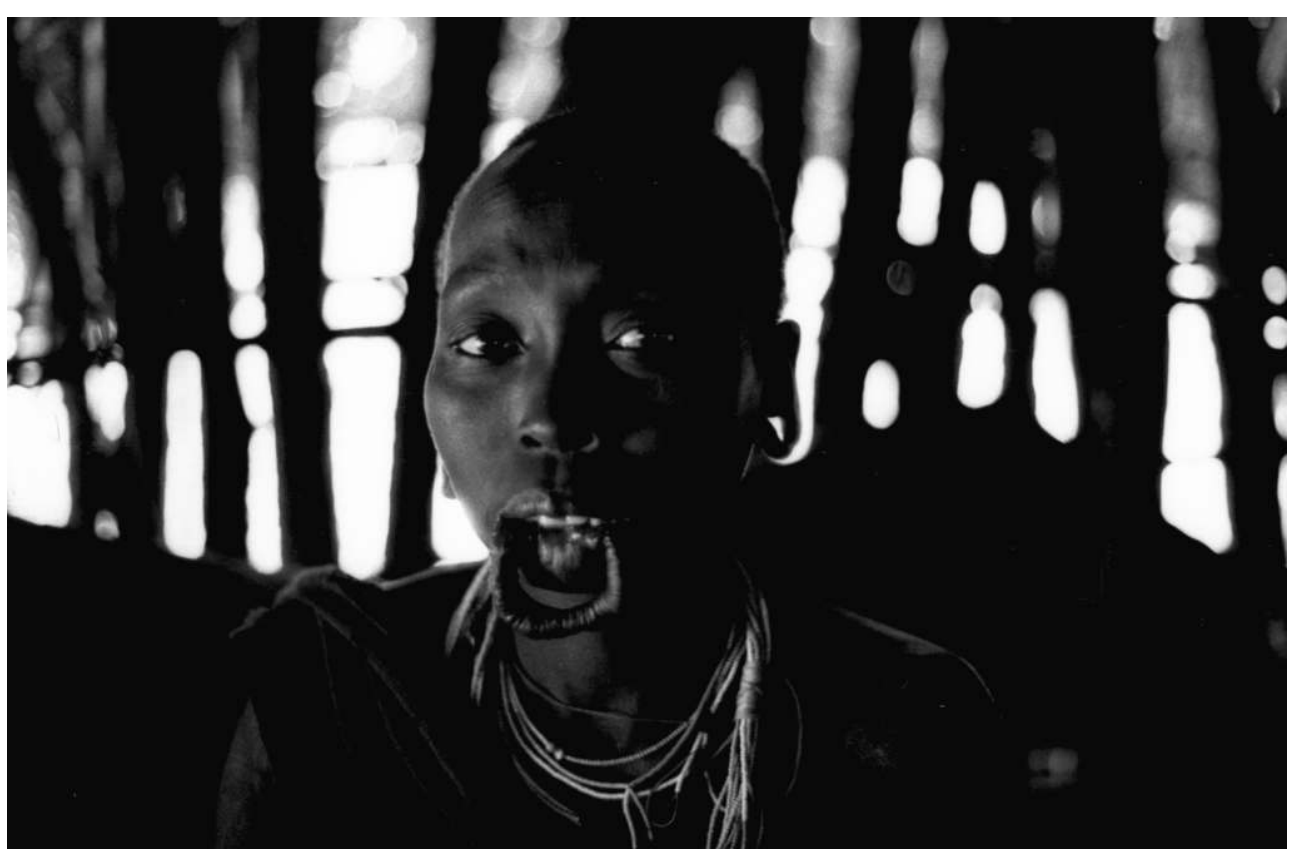

Femme Suri (voisins Est des Mursi) sans son labret. Kibish, 2007 (photo : J.-B. Eczet)

14 À l'échelle de l'existence, le labret est porté de la maturité sexuelle à la naissance des premiers enfants, c'est-à-dire pendant quelques années seulement. Les femmes le délaisseront ensuite et la lèvre se rétractera. À l'échelle quotidienne, le port du labret est réservé à certaines occasions, comme on l'a vu plus haut. Les femmes Mursi sont donc le plus souvent sans labret et la cicatrice se dévoile inévitablement. Je n'ai pas eu l'occasion d'observer comment se passe le perçage de la lèvre mais dans tous les cas, décider de se percer la lèvre et se percer la lèvre est une affaire de femmes. En plus du labret, des scarifications dites kichoa, réalisées sur le ventre des femmes, sont indispensables, moins au moyen d'une prescription ou de la peur d'éventuelles 
sanctions que par le consensus général autour des conséquences d'un manquement : la maladie ou la mort guetteraient la femme non scarifiée ou son enfant. La femme arbore donc une "parure de scarifications" avec le trou du labret et les scarifications ventrales. Pour bien saisir les raisons de cette double cicatrice sur le corps des femmes, il convient de faire un détour par les scarifications en général chez les Mursi, et d'introduire l'analyse par la crainte récurrente du sang qui coule.

J'étais assis avec Olekiwo, le frère de mon hôte, lorsque qu'une ancienne amie de sa mère vint lui parler de son comportement, toujours combatif, parfois trop. Elle s'en moquait gentiment et le mimait tapant dans tous les sens lors d'un duel au bâton. Elle prolongea son imitation en utilisant les gestes des duels féminins où de lourds bracelets métalliques prennent la place des bâtons de bois, jusqu'à ce que, par inadvertance, elle frappa avec un peu trop d'enthousiasme Olekiwo à la tête, qui saigna. Aussitôt, le jeu s'arrêta et elle appela un jeune garçon qui lui ramena de la cendre. Elle n'en mit pas sur la plaie mais sur le visage d'olekiwo et le sien, ainsi que sur tous les témoins de la scène. Un autre épisode, une dispute générale dans le campement où je me trouvais, se solda par plusieurs blessures sanguinolentes et donna également suite à un recouvrement collectif du visage avec de l'argile. Les Mursi disent que se recouvrir de cendre ou d'argile est un moyen de se protéger d'un danger. D'un point de vue pragmatique, on peut y voir la réaffirmation d'un lien, au moyen de la peinture, entre des personnes mises en péril ${ }^{11}$. Cette réaction face au danger est fréquente, que ce danger prenne la forme de cauchemars, de sorcellerie ou de maladie. Et le sang qui coule, même superficiellement, est toujours traité comme un danger. D'une manière générale, on ne peut pas contrôler la plupart des évènements où le sang coule : la mort, les blessures, la maladie, les menstruations, l'accouchement et, on l'a vu, la blague qui a mal tournée. Par contre, certains saignements sont récurrents et le danger qu'ils représentent est prévisible. Le problème initial du danger des saignements récurrents ${ }^{12}$ est ainsi le dénominateur commun à la prise d'initiative qui aboutit aux scarifications : en faisant couler le sang de manière volontaire et contrôlée, les Mursi en reprennent l'initiative. Ainsi, chaque type de scarifications est une prise d'initiative sur un danger spécifique.

\section{Marques initiées}

Les kichoa peuvent être effectués par tout le monde sans distinction de genre et il est fréquent de voir un motif en double $U$ inversé sur le deltoïde et de longues lignes sur les flancs. Par contre, celles sur le ventre sont exclusives aux femmes et perçues comme nécessaires à leur ontogénèse. Elles sont réalisées en plusieurs étapes tant la zone couverte par les scarifications est étendue. Trois bandes horizontales suivent les muscles abdominaux tandis qu'une bande verticale partage le ventre en deux. Chaque bande est constituée de trois lignes, elles-mêmes réalisées en tiretés. Chaque tireté est une cicatrice chéloïde obtenue au moyen de deux coups de rasoirs (autrefois de l'obsidienne). Un bout de peau est soulevé avec une épine et deux incisions sont données à la partie tendue avec un angle un peu supérieur à 90 degrés. A la fin de la réalisation d'une bande, les cicatrices à vif sont rincées avec de l'eau puis recouverte de charbon ou de cendre. Les séances de scarification s'inscrivent dans la continuité des actions quotidiennes. Elles ont lieu quand la femme est prête et qu'elle en a fait la demande à une spécialiste, qui n'a de particularité que d'être habile de ses doigts. Lors de mon observation, Luke travaillait aux champs avec sa coépouse et une autre femme $\mathrm{du}$ village. Ces deux dernières continuèrent leurs activités, l'une s'occupant de ses 
enfants, l'autre triant les graines de sorgho. Un groupe de trois jeunes filles passèrent non loin et vinrent s'arrêter, opportunistes, pour regarder. La spécialiste était venue avec une de ses meilleures amies qui vivait aussi dans le village de Luke. Bref, rien ne laisse supposer un changement de contexte particulièrement saillant tant au niveau des acteurs en présence que dans le cours des activités. Le seul comportement faisant l'objet d'une prescription particulière est que la femme en train d'être scarifiée doit rester calme en ne manifestant pas sa douleur par des gémissements ou des pleurs. Luke resta ainsi presque impassible, laissant même échapper un rire vers la fin, un peu crispé. Les premières bandes sont réalisées au début de la puberté et le motif complet peut être achevé des années plus tard, comme pour Luke qui a certainement dépassé vingt ans (fig. 5).

Fig. 5

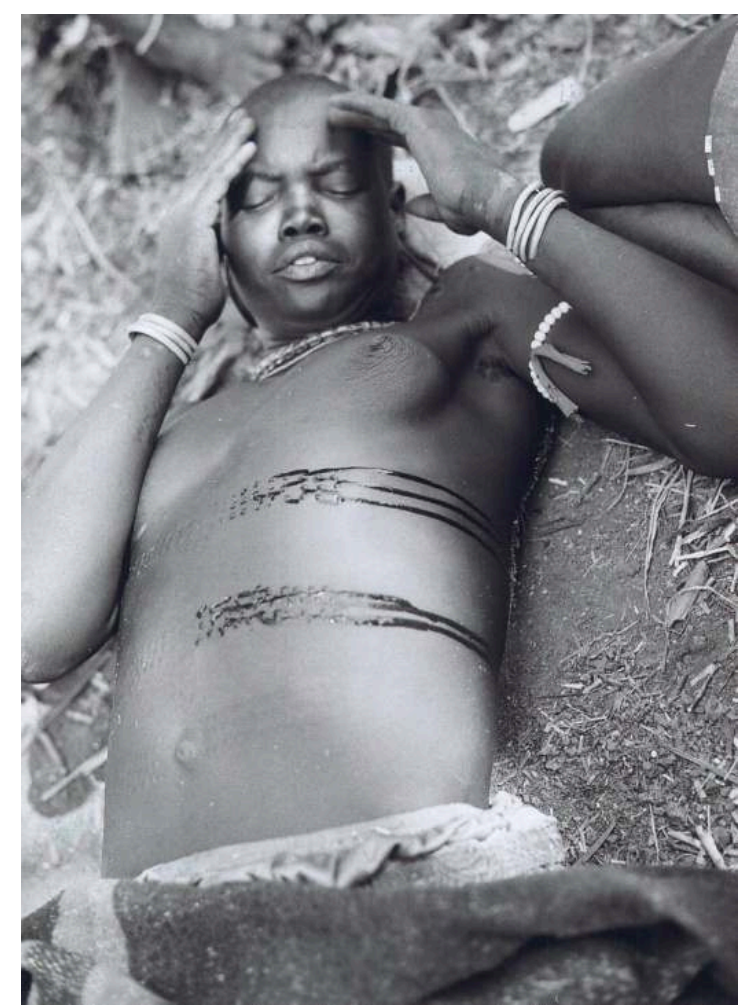

Luke lors de sa dernière séance de scarification. Elle était enceinte quelques mois plus tard. Mako, 2008 (photo : J.-B. Eczet)

Bien qu'il soit recommandé d'avoir le ventre scarifié avant d'être enceinte, sous peine de rendre le nouveau-né malade ou de faire une fausse-couche, ceci reste au conditionnel. Il n'y a pas de prescription stricte et leur réalisation reste in fine à la discrétion de chacun ${ }^{13}$.

D'autres cicatrices, imputées aux «pères ${ }^{14}$ » lors de corrections quotidiennes ou rituelles sont aussi appelées kichoa. Il est fréquent d'en apercevoir sur le sternum, les épaules et le dos des hommes. Elles alternent avec celles provoquées par les duels aux bâtons dits donga, infligées cette fois par les " paires » et elles ont évidemment, dans la taille comme dans la forme, l'indétermination du coup porté à la volée.

19 Toutes les kichoa semblent avoir la même appréciation, des commentaires minimaux, positifs mais sans emphase : c'est bien (a challi), j'ai juste coupé (anye kedi hon'), il m’a 
frappé (kadakto). Bien que ce soit des marques valorisées et jamais dites disgracieuses, on ne s'en vante pas, même de celles issues d'un combat ou d'une scarification volontaire. La proximité des commentaires associés et l'homonymie entre ces marques, pourtant différentes par le motif et par leur origine, témoignent d'une nature commune : la forme cicatricielle est le résultat standardisé d'une réaction à différents évènements. Car au-delà de l'apparente dissemblance entre les évènements à l'origine de ces cicatrices, c'est bien un même type de problème qui se retrouve, celui du sang qui coule de manière récurrente.

\section{Inscrire le volontaire dans l'impondérable}

En effet, les combats au bâton dits donga ${ }^{15}$ sont l'équivalent interne aux conflits engageant les Mursi et leurs ennemis ${ }^{16}$ : des jeux de guerre tout à fait sérieux mais dont le risque est contrôlé - il ne faut surtout pas tuer. Ces combats, provoquant le sang qui coule et les cicatrices, ne peuvent se faire qu'en période de bonnes récoltes. Si des menaces de famines ou de maladies planent sur le pays, alors le donga est interdit par les « anciens » détenteurs du pouvoir symbolique (classe d'âge bari). Le sang est un luxe (une prise de risque) qu'on ne peut faire couler que si le reste va bien. En d'autres termes, on ne peut dans ce cas en prendre l'initiative qu'à la condition que la mort, la perte et la faiblesse soient loin, que la situation actuelle reflète l'opulence et la stabilité. Par contre, le meurtre d'un ennemi - quand le sang d'un « Autre non Mursi » a coulé -, fait l'objet d'une scarification "sèche ". Appelée riru, cette marque est le fruit d'une brûlure. L'initiative sanguinaire n'a pas besoin d'être située sur le corps d'un Mursi en raison de la nature différente de l'ennemi. Cette cicatrice n'est ni compensatrice, ni initiatrice, car le sang coulé n'était pas endogène et on ne tire que le prestige de la marque (non obligatoire, d'ailleurs). La contrepartie du saignement n'est pas recherchée vis-à-vis de quelque chose qui ne nous concerne pas.

Peu importe l'échelle (le pays ou la personne) il faut être concerné. Les kichoa que tout le monde peut faire à sa guise sur ses épaules, son dos et ses flancs sont parfois précédés d'un épisode pathologique. On en voit d'ailleurs certaines sur des enfants assez jeunes qui d'habitude ne sont concernés par aucun des évènements précités (combats, meurtre, menstruation, accouchement) et qui sont justifiées par une maladie passée. La plupart des kichoa, unisexes et volontaires, sont très pratiquées chez les jeunes hommes et les jeunes femmes. Hommes comme femmes inscrivent ainsi, pendant quelques années des motifs sur leurs corps qui soulignent le plus souvent l'anatomie. Se confronter à l'ethos guerrier et aux actions à faire pour s'y conformer pousse à faire couler son propre sang. Ces jeunes gens qui pratiquent le donga, ou le ula pour les femmes, anticipent sur leur propre corps le sang qui coulera du corps de l'autre. On voit donc un glissement et un enchâssement : de la cicatrice " sèche » due au sang de l'étranger qui n'en appelle pas d'autre, on trouve la cicatrice subie lors d'un donga, un sang personnel qui coule à condition que le collectif se porte bien. Ontocentrées, les kichoa volontaires sont la contrepartie au sang que chacun s'apprête à faire couler chez d'autres Mursi ${ }^{17}$. Luxe que l'on peut se permettre, compensation du coup provoquant le sang ou miroir volontaire de la maladie, ces kichoa imitent ou anticipent l'aléatoire subie du sang qui coule. 


\section{Sang pour sang femme}

22 Le visage d'une femme, qui a, par défaut, la lèvre pendante, est indissociable de son ventre scarifié. Cette parure de scarification vient ponctuer ces transformations féminines du corps qui sont subies et que le sang vient ponctuer: les menstruations, l'activité sexuelle à son commencement et l'accouchement. C'est pourquoi elles sont les plus impératives: à la différence d'un coup portée volontaire ou d'une maladie éventuelle, ces étapes récurrentes sont le lot commun et inévitable de la physiologie féminine. Les marques ventrales sont l'initiative féminine sur la procréation comme la lèvre qui se perce est l'initiative féminine sur l'activité sexuelle au début de la puberté. La manière de faire couler le sang - percer, couper - comme le lieu renseignent par analogie sur la nature de ces évènements proprement féminins- défloraison, menstruation et procréation. Le traitement par la scarification vient les inscrire dans les corps et dans les actions volontaires des personnes ${ }^{18}$.

Il faut aussi noter que ces marques ne sont pas permanentes. Au même titre que les thèmes qu'elles expriment, elles disparaissent quand leur mention n'est plus de mise. Les femmes âgées qui ne portent plus le labret voient leur lèvre se rétracter et la bouche se reformer. De même, le vieillissement cutané, amplifié par les grossesses successives, fait peu à peu disparaitre les scarifications ventrales. À mesure que les grossesses appartiennent au passé et que l'activité sexuelle diminue, les scarifications s'effacent.

Enfin, si l'on peut concevoir ces deux actes comme une étape initiatique, il faut alors entendre initiation au sens actionnel du terme. Et quand David Turton dit très justement que « the lip-plate worn by Mursi women is an expression of female social adulthood and reproductive potential ${ }^{19}$ ", il ne faut pas le comprendre comme un statut, celui d'initié ou d'épouse, qui est conquis mais comme des pratiques qui engagent chaque femme dans sa condition ${ }^{20}$. La faible complexité relationnelle de la séance de scarification, qui est au contraire souvent riche dans l'initiation ${ }^{21}$, témoigne de l'expérience individuelle qu'elle propose. Initiée par la personne elle-même, c'est une douleur singulière et peu transmissible sur un support inaliénable que produit cette intériorisation du point de vue des femmes sur elles-mêmes. On complétera donc une proposition précédente en définissant ce que pourrait être les scarifications en générale chez les Mursi, à savoir qu'elles sont issues d'un même problème initial (sang qui coule) qui se retrouve dans différents évènements (combats, maladie, physiologie) traités sous une forme commune (découpe de la peau et saignement) de manière individuelle (douleur personnelle) faisant l'objet d'une sanction sociale (trace visible de la scarification) (fig. 6). 
Fig. 6

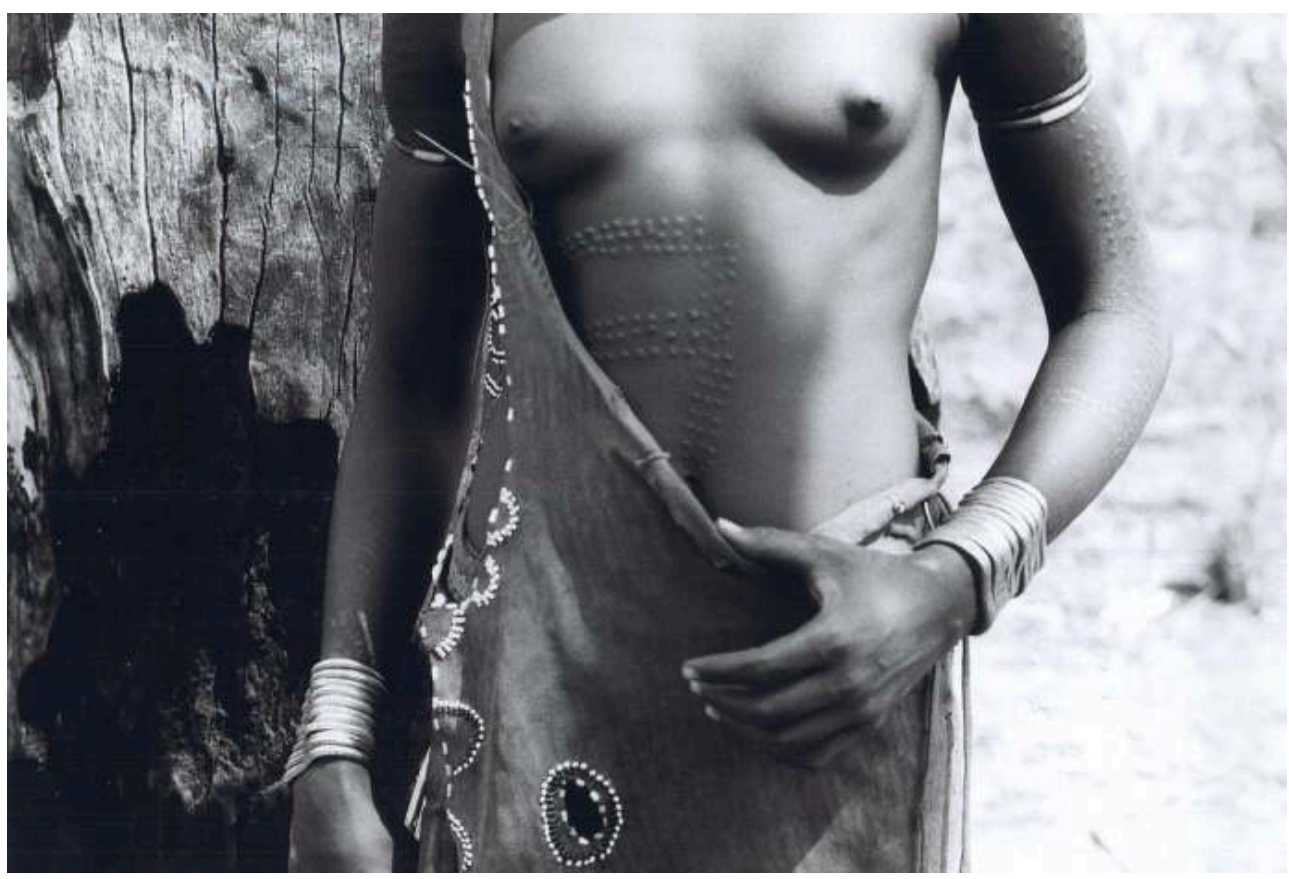

Scarifications ventrales dont il reste plusieurs bandes à réaliser pour que le motif soit complet, Mako, 2008 (photo : J.-B. Eczet)

En bref, les cicatrices ventrales et labiales est un point de vue des femmes sur ellesmêmes par la prise d'initiative sur les évènements proprement féminins du corps : perte de la virginité, menstruation et procréation.

\section{En passant par la femme cosmos}

Jusqu'à présent, la femme s'est mue autour du labret puis s'est trouvée marquée par la trace qu'il laisse dans la chair. Or, le labret est aussi un objet avec une forme spécifique. S'il ne prend tout son sens que dans son intégration au corps, en présence ou en creux, sa matérialité informe aussi d'un autre usage du corps des femmes.

En pays Mursi, peu de formes visuelles rappellent explicitement les activités humaines ${ }^{22}$. Le contexte environnemental des Mursi est composé d'une savane arborée qui alterne avec des plaines herbeuses où la présence humaine n'est visible que dans les villages. Les quelques chemins qui les relient parfois diffèrent peu de ceux crées par les animaux autour des points d'eau et il faut le plus souvent traverser les prairies d'herbe dès lors qu'on se déplace à travers le pays. Hors du village, les Mursi ne modifient pas l'apparence du monde d'une manière contrôlée qui aboutirait à des formes géométriques, comme pourraient l'être des rues, des terrassements, des cimetières, etc. Ils rejettent eux-mêmes tout ce qui se trouve à l'extérieur des zones d'habitation dans la catégorie souvent péjorative de gasho (savane ou bush). Les champs (gui, pl. guyo) euxmêmes ne répondent pas de formes spécifiques ni dans la délimitation des parcelles, souvent déterminée par le sol, la végétation et leurs contraintes, ni par les rythmes de plantation du sorgho, où les nouveaux plants sont disposés «suffisamment loin » des autres, sans rangée. L'aléatoire visuel ne propose pas de rythmes sur lesquels s'appuyer ni d'événements récurrents par lesquels on puisse être saturé. Il suffit que le lecteur de 
cet article lève les yeux sur son environnement actuel pour qu'il découvre le rectangle de son écran ou de son livre, de ses feuilles à l'unique proportion du format $\mathrm{A}$, de sa table et de la pièce dans laquelle il est, des lignes droites des couloirs, des piliers et des rues, et les rythmes données par la multiplication de ces formes, touches du clavier, cases des bibliothèques, rangées des livres et des fenêtres, des tables et des chaises, escaliers et enchainements des rues, pour s'apercevoir que notre vision est quant à elle saturée de formes géométriques et de volumes - qui tous ont pour base le rectangle qui, justement, à trop se répéter, s'oublient. L'environnement visuel des Mursi ne propose pas ces stimulations spécifiques permettant de ce fait de repérer certaines formes avec davantage d'acuitée 23 .

Le labret n'a pas d'équivalent ni en texture ni en bord ${ }^{24}$ et contraste avec l'environnement perceptif habituel. Ce disque est une forme originale par ses caractéristiques propres de régularité, et son lieu de mise en valeur, dans le visage des femmes, a pour effet immédiat d'augmenter encore sa saillance perceptuelle. Isolé dans le continuum aléatoire des perceptions, ce modèle géométrique se retrouve dans les activités humaines de type cérémoniel : cercles concentriques de pierres qui se forment à mesure que les sacrifices de vaches ont lieu, circulation du bétail lors des bénédictions, disposition spatiale des individus lors des danses et des déclamations de poèmes, etc. À cela, il faut ajouter la forme circulaire de l'habitat. Tous les moments collectifs cérémoniels ${ }^{25}$ prennent donc la forme d'un cercle construit par les acteurs, les spectateurs et leurs dispositifs (les pierres) - et même au-delà des personnes en présence, les dômes formés par les huttes, signe par excellence de la présence humaine (fig. 7).

Fig. 7

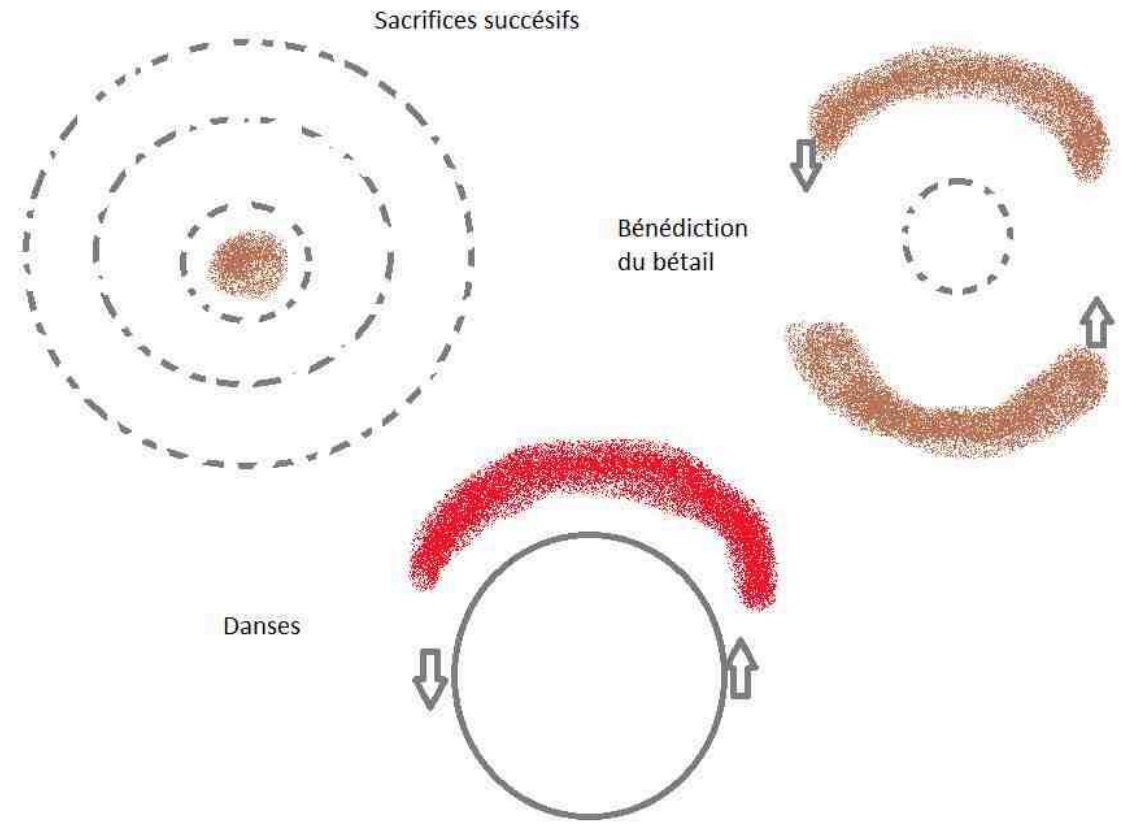

Différentes configurations spatiales de processus rituels sur le modèle du cercle (graphique : J.-B. Eczet) 
Mais tout cela est éphémère. Lorsque la danse s'arrête, lorsque le rituel ou les chants de poèmes se concluent, lorsque le bétail s'en retourne, lorsque les Mursi suivent les transhumances et quittent leurs huttes qui s'écroulent et lorsque, enfin, l'herbe repousse et cache les pierres, la saillance perceptive du cercle s'évapore. Si le cercle est la forme que prend la présence humaine en collectivité, alors il est aussi éphémère que ces réunions et disparaît aussitôt que les intérêts et les activités de chacun s'entrecroisent et ne convergent plus. À ces formes dispersées dans l'espace et dans le temps, le labret oppose la permanence en s'implantant dans le corps qui, lui, suit les déplacements des personnes dans le temps et dans l'espace. Le cercle n'a ainsi pas besoin du trop rare collectif pour exister. La femme arbore une forme sous déterminée, archétypale, qui figure l'activité humaine collective. Il s'agit d'un affichage et non d'un simple bout d'argile en ce qu'il "est une surface qui a été configurée ou traité de manière à exhiber une information irréductible à celle de la seule surface ${ }^{26}$ ", malgré son indétermination et son absence de signes. C'est un modèle saillant de l'espace visuel disposé dans le visage de manière à le rendre encore plus visible et qui concentre, en un tout délimité, ces activités humaines et leur variabilité. Le labret n'est pas un aide-mémoire de la forme que ces activités de groupe doivent prendre. Le labret est plutôt ce qui lie la vie individuelle et collective en gardant dans le champ perceptuel quotidien une partie de ces formes collectives. Et lorsque les Mursi s'en vont hors de leur territoire, c'est-à-dire lorsque l'identité mursi est mise à l'épreuve, le labret n'agit pas comme un emblème distinctif à la manière d'un blason qui raconte une histoire ou une symbolique des valeurs, mais comme le point de départ de ce qu'être Mursi veut dire, moins la connaissance d'un corpus symbolique que la participation à certaines pratiques. Les femmes ornées garantissent par leur présence l'actualisation de cette modalité mémorielle : garder en vue ce que nous faisons ensemble ${ }^{27}$.

Depuis peu, une autre ornementation corporelle reprend aussi cette forme. Les Mursi n'apprécient pas les cheveux longs et c'est autrefois avec de l'obsidienne qu'ils se rasaient la tête. Cet outil ne permettait pas une grande finesse dans la coupe et les quelques documents photographiques anciens montrent des cheveux qui ont anarchiquement poussés sans recherche de motif quelconque ${ }^{28}$. C'est avec l'accès récent aux rasoirs métalliques que les Mursi peuvent développer des coupes de cheveux plus élaborées. Celles-ci sont variés mais déclinent toutes un même motif : la coupe minimale est un disque sur le sommet du crâne (cherrita) auquel s'ajoute deux bandes horizontales, l'une près du front, l'autre près de la nuque (shulshule). Parfois même des cercles concentriques sont réalisés atour du disque central (fig. 8). 
Fig. 8

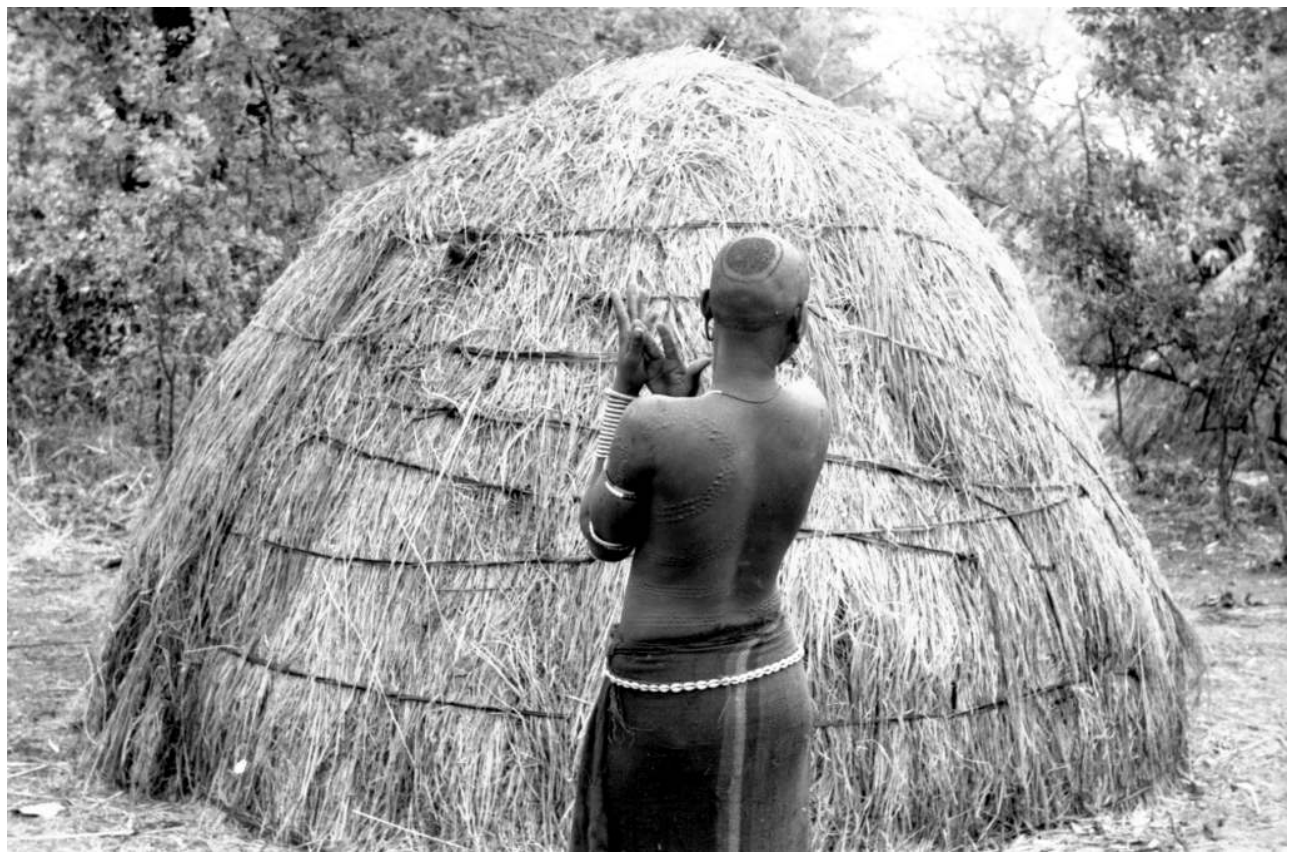

Cercle de la cherrita et de la hutte (dori, pl. doren). Mako, 2008. (photo : J.-B. Eczet)

Comment ne pas voir dans ceux-ci la référence aux sacrifices où les cercles sont faits de pierre, comme dans les bandes de cheveux de la nuque et du front, les troupeaux de bétail qui tournent plusieurs fois autour du prêtre (komoru) les bénissant, lui-même au centre d'un cercle de pierre (fig. 9)?

Fig. 9

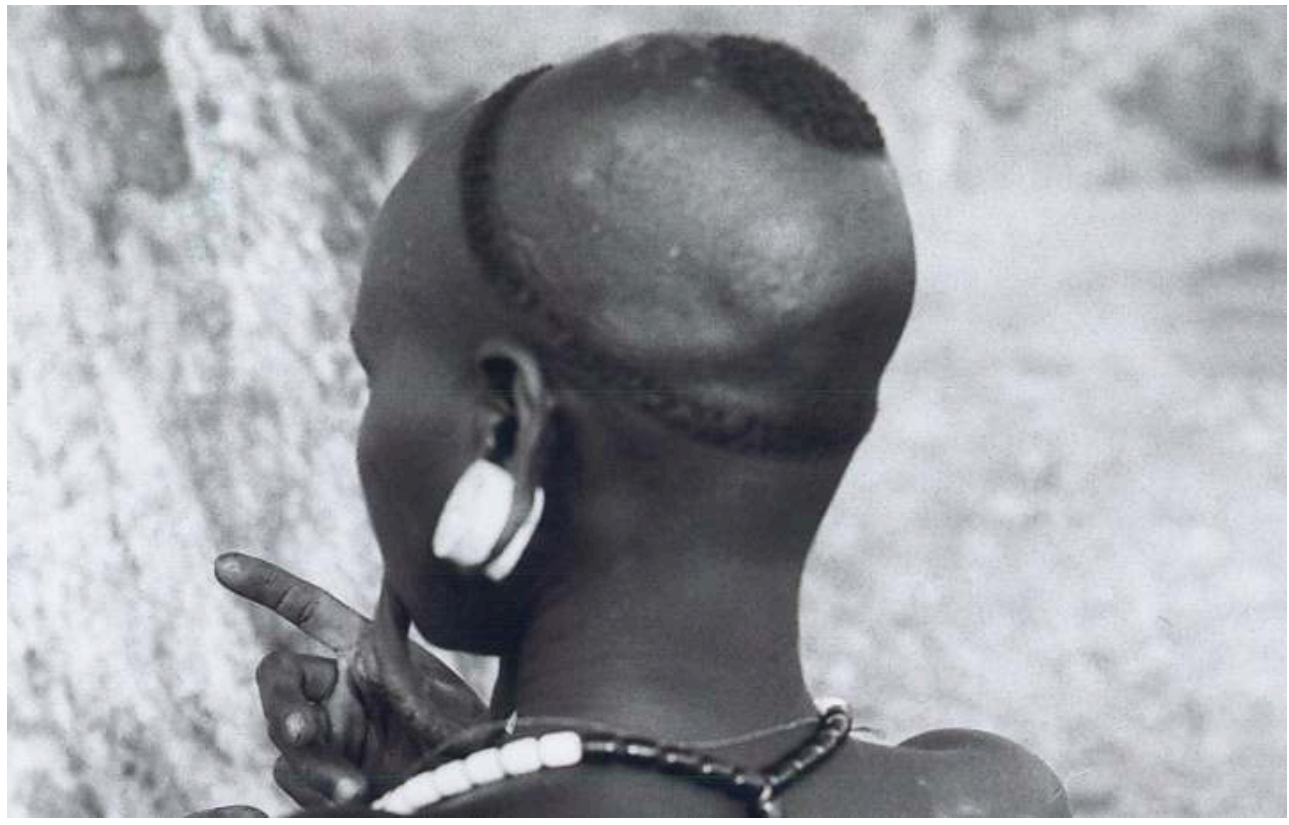

Le cercle sur le sommet du crâne est le plus souvent le motif minimal auquel on ajoute deux bandes (une sur la nuque, l'autre près du front) ou un cercle, comme sur cette photo. Mako, 2008 (photo : J.-B. Eczet) 
Il faut aussi noter que les débats publics, lieu d'expression collectif du politique dans ces sociétés à classes d'âge sans chef institutionnalisé, ne suivent pas le modèle du cercle. Ceux-ci s'en distinguent nettement : une assemblée est assise faisant front aux orateurs qui alternent. Ces derniers donnent leur point de vue sur la situation qui est à l'origine de la tenue du débat, marchant en ligne droite le long de l'assemblée, un bâton ou une kalachnikov à la main, et multipliant les allers-retours jusqu'à la fin de la prise de parole. Dans ce cas, le cercle n'est pas de mise car le consensus social, s'il s'exprime tout de même par le respect de la procédure, n'est pas de même nature que durant les danses ou les sacrifices. Ici, un problème d'avis contradictoire est à l'origine de la réunion et les participants ne s'accorderont peut être pas sur la suite à donner. Pis encore, de ces débats pourront éclore des envies de scissions et des querelles renforcées. Bref, le politique n'est pas sur le mode du consensus apriori, à l'inverse des autres activités collectives déjà mentionnées. Le cercle est la forme que prennent des choses indiscutables.

\section{Sirènes de l'Omo et vaches de femmes}

Défiguré, au sens littéral du terme, n'est pas péjoratif mais désigne que la figuration ici, le visage - est détruite ou déformée. Le labret positionné, avec ceux situés dans les oreilles qui ajoutent des volumes et des plans que les lobes à eux seuls ne fournissent pas, on découvre un visage aux proportions inhabituelles, loin du canonique trait sourcillaire, séparant horizontalement la face en deux parties égales et probablement un peu déroutant pour un système cognitif de reconnaissance des visages ${ }^{29}$.

Se pose donc l'inévitable question de l'esthétique de cette pratique. On pourrait poser la question sous la forme relativiste : «À quelle esthétique répond-elle? » considérant que son appréciation positive est le fruit d'un apprentissage arbitraire comme on pourrait chercher un équilibre et une justesse perceptuelle dans l'objet "visagelabret » reconnaissable par tout un chacun. On risquerait alors de tomber dans le piège linguistique en substituant à "esthétique » le terme "beau ", institué en concept qui navigue entre son acception adjectivale et nominale ("c'est beau » et « le beau»), de l'appréhension d'un ressenti à la définition d'une propriété des $\operatorname{choses}^{30}$. Cette alternance entre des affects (émotions) et la cause des affects (objet) peut faire perdre de vue ce qu'on entend par esthétique sans qu'on recherche les raisons qui font dire que le labret est quelque chose d'esthétique soit par essence, soit par apprentissage arbitraire. À la recherche de ce qui fait que le labret est un objet « ontologiquement esthétique », on lui préfèrera celle qui veut montrer en quoi il est « constitutivement esthétique $^{31}$ ». Dans ce cas, il s'agira de décrire ce qui donne l'appréhension positive de cet objet en tant que «signal particulièrement couteux ${ }^{32}$ » dans un environnement perceptuel donné (celui des Mursi).

Ainsi, au-delà du corps qui se meut de manière adéquate, qui se marque pour avoir l'initiative d'un danger et qui porte une forme géométrique nodale, il faut donc considérer ce visage littéralement défiguré au moyen du labret afin de comprendre tout à fait l'appréciation positive "c'est bien", dont on ne sait pas encore si elle s'adresse aussi au résultat sur le visage.

Pour appuyer mon dernier argument qui prolonge le précédent en pointant aussi des ressemblances formelles, il me faut décrire brièvement un domaine connexe où les 
analogies sont aussi riches qu'évidentes. La praxis mursi des perceptions visuelles se caractérise par la recherche d'analogies des couleurs et des motifs autour d'un point focal, le bétail. La vie des Mursi et celle des bovins sont imbriquées et aucune activité humaine ne peut se dérouler sans la présence réelle ou référentielle du bétail. Or, ce dernier est désigné en fonction des couleurs de sa robe, de même que les humains sont désignés et nommés au moyen des couleurs de ces robes bovines (fig. 10).

Fig. 10

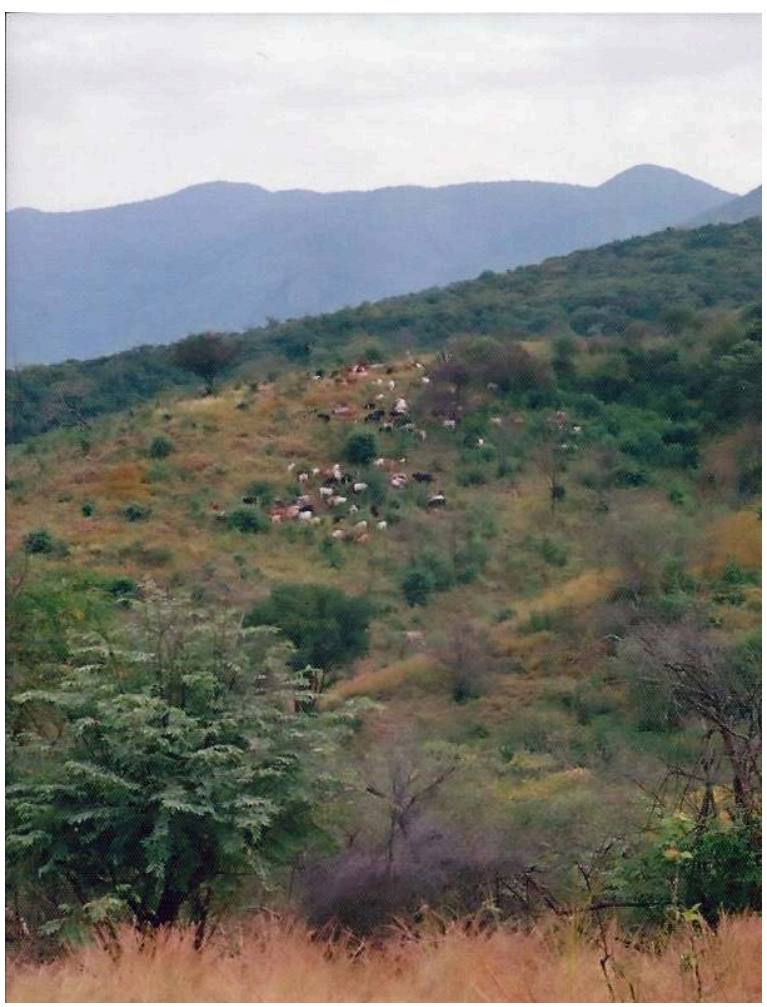

Les robes du bétail Mursi sont variés et les plus récurrentes constituent les taxons de leur classification des couleurs. Kutula Tula, 2007 (photo : J.-B. Eczet)

Donc, parmi les propriétés qu'ont les individus, celle d'être d'une couleur en est une fondamentale. Il s'agit autant de cattle complex ${ }^{33}$ que d'un colour complex ${ }^{34}$, en ce que les humains, le bétail et leurs relations utilisent la couleur pour prendre forme. Le premier nom d'un homme a la forme "taureau + couleur", comme dans Taureau Noir ou Taureau Rayé, puis tous les autres noms seront des déclinaisons de cette couleur originelle. Les déclinaisons des couleurs dans les noms proviennent du fait que les Mursi particularisent les relations jusqu'à attribuer une identité spécifique à chaque personne selon la relation vécue. Les noms sont ainsi des variations de la couleur fondamentale des individus et indexent chacun une relation duale. Chaque individu a donc potentiellement autant de noms que de personnes avec qui il interagit. Ces couleurs déclinées sont déterminées par les robes de vaches les plus récurrentes et les noms sont créés en cherchant dans les perceptions visuelles ce qui les rappellera : pour la robe noire (koroy), on utilisera les scarabées (gule), les fourmis (gona), le buffle (nebi), la pleine nuit (barkiango), l'ombre (rii), et toute ambiance qui le rappelle. Pour le blanc (holi), ce sera par exemple le plumeau des roseaux d'eau (bale) ou encore le soleil et sa lumière zénithale aveuglante (su kale). Pour la robe de vache " tacheté rouge " (kori), on mobilisera la girafe (kirin) ou on créera des choses de cette couleur, comme dans le nom 
Singe Tacheté Feu (Kalamdonigo), et ainsi de suite. Les fondements identitaires et nombre d'interactions font ainsi appel, au moyen de l'anthroponymie et des poèmes chantés, à des perceptions visuelles et aux analogies qui y sont repérées ${ }^{35}$.

Les fondements identitaires sont largement déterminés par le bétail et, en conséquence, l'appréhension positive de la forme plastique « bétail » est un attracteur qui pousse à rechercher des formes plastiques qui s'y rapportent. Car il n'y a pas de raison a priori pour que ces fondements qui se rapportent au bétail et se traduisent dans des formes rhétoriques ne trouvent pas aussi des formes plastiques dans lesquelles s'exprimer: parce que, d'une part, les humains se construisent au moyen d'analogies formelles entre les choses du monde dont le bétail est un index vers lesquelles les comparaisons reviennent toujours; et parce que, d'autre part, le bétail n'a pas d'équivalent chez les humains ni par son apparence ni par sa psyché et qu'il ne s'agit pas seulement pour ces derniers d'imiter le premier. La plastique trouve des formes intermédiaires entre ce qu'est un bétail non modifié par l'humain et une femme non modifiée par l'humain. Personne ne copie l'autre au point de devenir comme lui, les collectifs et les formes plastiques se nourrissent mutuellement ${ }^{36}$.

Les modifications des corps humains et bovins se reflètent : les scarifications des uns se voient sur les corps des autres, les peintures sont communes et souvent réalisées dans un même élan sur les deux corps, et les bijoux sont de même facture. Le labret participe à réduire la distance entre forme des visages et forme des faces. Aux femmes, il manque le mufle comme aux vaches manque la rondeur du crâne. Le labret change les proportions du visage féminin qui s'approchent alors de celles du bovin. Ce dernier a la forme de ses cornes modifiée au moyen du travail des humains qui frappent la base des cornes avec de lourdes pierres pour en modifier la courbure. Celle qui est appelée chipto (noué) revient sans cesse et dessine la silhouette du crâne bombé qui manque aux bovins (fig. 11). 
Fig. 11
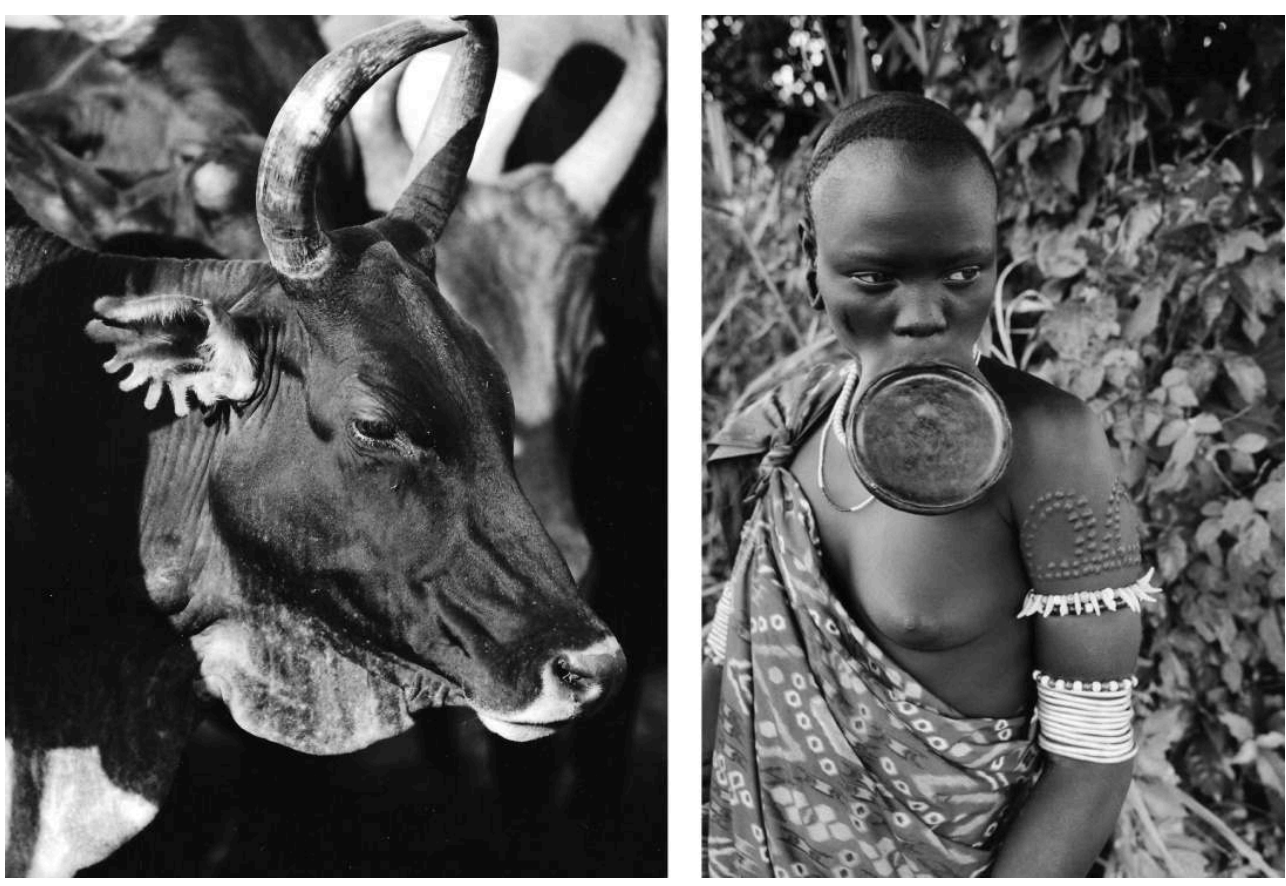

Le bétail voit son corps modifié en miroir avec celui des humains : les pointillés des oreilles sont des scarifications et rappellent les tiretés des kichoa, et la forme des cornes transforment les proportions de la face, comme le labret changent les proportions du visage. Mako, 2008 (photo : J.-B. Eczet)

Lors des danses, la gestuelle modifiée des gestes quotidiens par le port du plateau n'est évidemment pas de mise et elle est remplacée par des sautillements rappelant ceux des veaux. Les jeunes femmes qui portent le labret rebondissent rapidement en souplesse et rappellent la jeunesse du bétail. Les hommes lèvent les bras pour représenter les cornes de leur taureau ou de leur bœuf et frappent ensuite la terre de leurs pieds pour imprimer dans le sol le bruit sourd des coups de sabots. À ces jeux de séduction s'ajoutent les femmes plus âgées qui, sans labret, traversent l'espace de danse en groupe, levant aussi les bras au ciel pour former les cornes - idiome gestuel élémentaire pour représenter le bétail -, et font entrer dans la chorégraphie générale le troupeau indifférencié. C'est ainsi que, dans les pratiques corporelles (ornementation et danses), les références identitaires s'entrecroisent. Mais on ne prend jamais l'un pour l'autre. Dans les ornements comme dans les danses, les imitations sont suggérées mais jamais complètes, fonctionnant davantage sur le mode de l'indexicalité que de l'iconisme. Les hommes et les femmes ne se peignent pas selon la couleur de leur bovin de référence ni ne se cachent sous des masques pour les imiter: l'identité n'est pas multipliée mais renforcée et affirmée en puisant dans des formes bovines. Les femmes ne sont pas des vaches, pas plus que les vaches ne deviennent des femmes. Il s'agit de formes valorisées issues de la proximité entre ces deux collectifs, dont l'appréhension esthétique positive est justement le fruit de ce rapprochement, de ce recouvrement partiel. L'identité trouve ici un autre niveau d'expression car le labret dessine une des figures les plus marquantes de l'esthétique mursi issue de la co-référentialité humains-bovins ${ }^{37}$.

41 Pour s'en convaincre, une comparaison locale s'impose. Les Nyangatom vivent au sud du pays Mursi. Egalement pasteurs nilotes, une différence importante entre ces deux groupes est leur rapport à l'eau, qui, dans cette région, prend la forme de ce grand fleuve nommé Omo par nous autres, Waro par les Mursi, Anam par les Nyangatom. Ces 
derniers, dont Serge Tornay a retracé l'ethnogenèse sur deux siècles ${ }^{38}$ (2001), ont émigré du nord de l'Ouganda, traversant les plaines kényanes désertes des Turkana jusqu'à se stabiliser dans une région à cheval entre l'Éthiopie et le Soudan. À leur arrivée, les berges du fleuve étaient occupées par un groupe peu nombreux, les Murle de l'Omo. Tornay montre comment ce groupe, disparu des récits au fil du temps, fut en fait largement décimé par une épidémie et les survivants assimilés par les Nyangatom ${ }^{39}$. Ceux-ci en gardèrent une division de la société en deux parties, dont l'une, plus sédentaire que les pasteurs nomades, entretient avec le fleuve un rapport de proximité immédiate et continue. Surtout, et c'est assez rare chez des nilotes pour être remarqué, les Nyangatom pèchent et mangent poissons et crocodiles, ce qui est aussi relevé péjorativement par les Mursi pour marquer leur différence. Bref, si les Mursi et les Nyangatom illustrent ce que Melville Herskovits nommait cattle complex, les derniers se différencient des premiers moins par les pratiques qui ont trait au bétail qu'en raison de la spécificité de leur rapport à l'eau, au fleuve et aux animaux consommés qui y vivent ${ }^{40}$.

Pourquoi ce détour par les Nyangatom et leur particularité alimentaire? Tout simplement pour appuyer le fait qu'en ces terres d'analogies formelles entre les choses du monde, il n'est pas étonnant de voir, sous la diversité des formes (ornements, corps, danses, etc.), certains schèmes esthétiques se retrouver. Et les Mursi diminuent la distance entre les femmes et les bovins comme les Nyangatom rapprochent formellement leurs femmes des êtres aquatiques. Une femme Nyangatom n'a pas la même silhouette qu'une femme Mursi en raison de sa tenue vestimentaire et de ses ornements. Ici, les proportions du corps humain sont malmenées pour s'approcher du canon des êtres aquatiques, poisson ou crocodile: les nombreux colliers des femmes font disparaitre leur cou sous un cône de perle. Il n'y a, entre le bas du visage et les épaules, aucun angle qui ne vient briser la continuité des plans. Le cou a disparu, la tête et le buste ne forment plus qu'un. La robe des femmes moule les fesses et le haut des cuisses pour finir évasée avec des franges de cuir de différentes couleurs. Enfin, le labret nyangatom qui, on l'a vu, est une langue de métal fixée sous la lèvre pouvant rappeler les barbillons des poissons chats de l'Omo, a souvent pour motif, ciselé dans le métal, des stries rappelant des écailles de poissons. Jonction des segments tête-corps remplie pour n'obtenir qu'une seule masse, robe prolongeant la continuité pour finir évasée et frangée, motifs d'écaille et barbillons, autant de traits qui rappellent la description d'un être aquatique ${ }^{41}$ (fig. 12, 13). 
Fig. 12

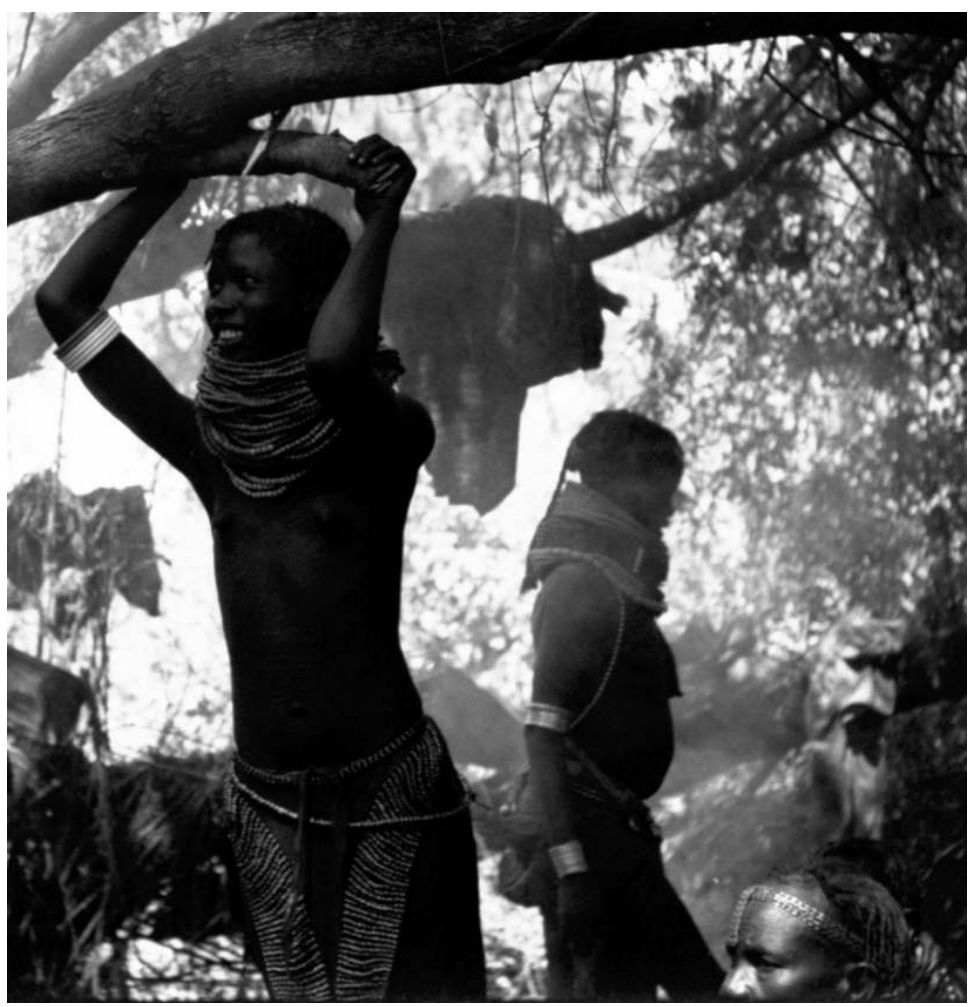

La transformation principale des proportions du corps féminin des Nyangatom se réalise au moyen des nombreux colliers de perles. Kangiten, 2007 (photo : J.-B. Eczet)

\section{Fig. 13}

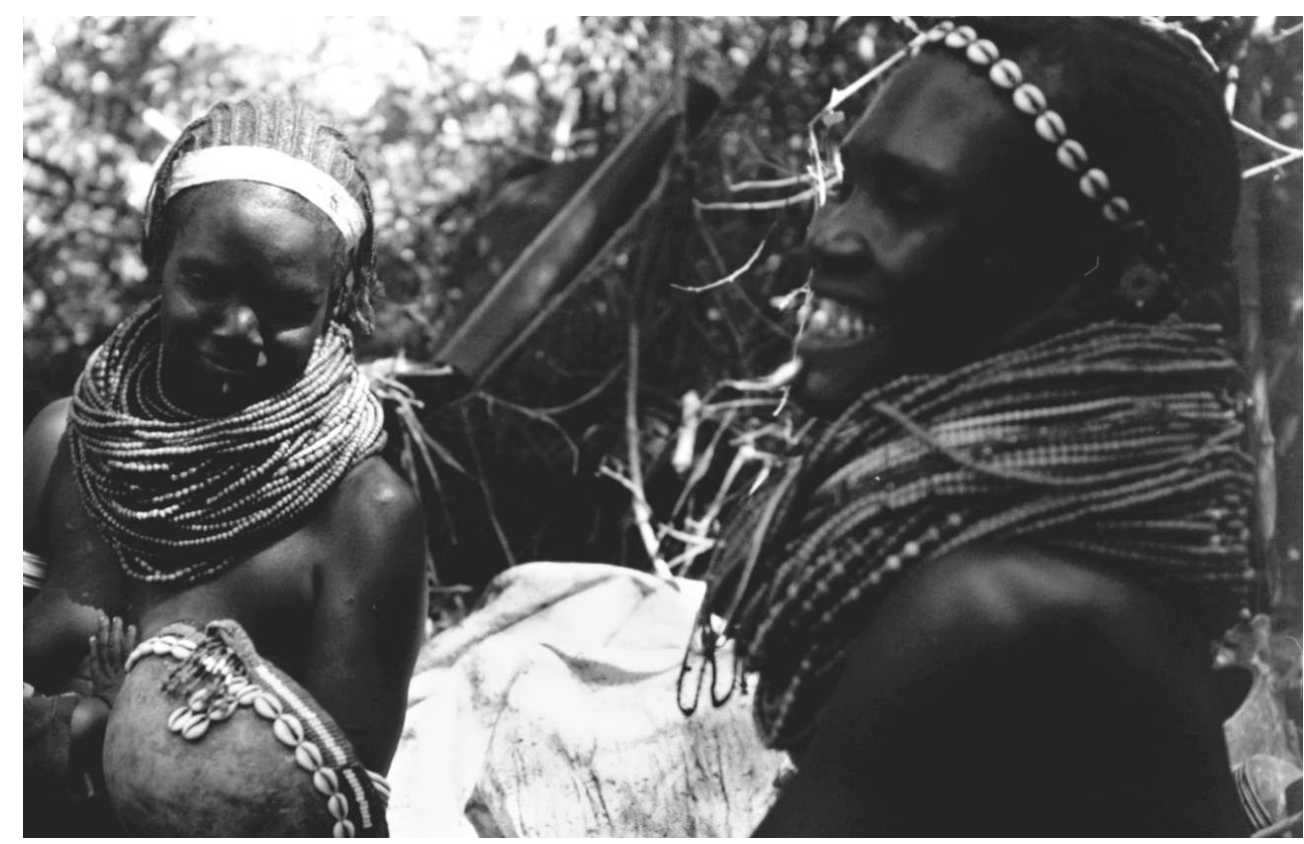

Les autres ornements (robes, labrets, coupes de cheveux, etc.) dressent aussi des analogies avec les êtres aquatiques en rappelant nageoires et écailles. Kangiten, 2007 (photo : J.-B. Eczet) 


\section{L'après labret}

Le labret agence un ensemble de points de vue et de relations cumulées entre les origines de ces points de vue sous la forme redoutablement efficace d'un simple disque d'argile ou de bois : point de vue des hommes sur les femmes, des femmes sur ellesmêmes, garantes de la permanence d'une forme sociale archétypale et, enfin, figures stéréotypées de l'esthétique mursi qui produit des formes intermédiaires entre les collectifs humains et bovins. Le labret est donc un objet à forte valeur identitaire, mais pas en ce qu'il signifie une appartenance. Le labret montre des relations de genre, de pratiques collectives et d'esthétique au moyen de différents contextes pragmatiques qui existent hors de lui et avec lesquels il s'associe: motion particulière, scarifications en générale, danses et rituels, ethos pastoral. C'est un chaînon commun à différents dispositifs et il réalise ainsi l'étonnant paradoxe d'être une synthèse puissante par tous les thèmes qu'il traite au moyen d'un dispositif simple. Mais c'est aussi sa sousdétermination qui en fait une des premières pratiques que des modifications relationnelles brutales font disparaître (missions, administration gouvernementale). Parce qu'il n'est pas un fondement nécessaire dont la disparition témoignerait d'un effondrement identitaire mais un point nodal dans un réseau d'idée et de relations, sa disparition ne bloque pas totalement un thème privé de sa réalisation et laisse libre cours à d'autres voies de formalisation.

\section{NOTES}

1. J'ai bénéficié, pour la rédaction de cet article, du prix de l'observatoire Nivea/CNRS et je tiens à remercier les membres du comité scientifique de l'Observatoire ainsi que tous les membres du jury.

2. Il s'agit des Padaung, du groupe Karen, vivant au Myanmar.

3. Wikipédia, http://fr.wikipedia.org/wiki/Mursi. Consulté le 14/05/2012.

4. Il n'y a pas de caste chez les Mursi et la dot est fixe et indépendante de la taille du labret (David Turton, "Lip-plates and "the people who take photographs" : uneasy encounters between Mursi and tourists in southern Ethiopia ", Anthropology Today, 20 (3), 2004, p. 3-8). Je cite une page de Wikipédia pour ne pas centrer ma critique sur une source en particulier mais on pourrait citer Angela Fisher et Carole Beckwith, African Ark, Harry Abrams, 1990 ; Gianni Giansanti, Afrique mystérieuse, Paris, Solar, 2004 ; Hans Silvester, Peuples de l'Omo, Paris, Editions de la Martinière, 2006.

5. Il est amusant de voir que la page Wikipédia sur les Padaung, les dites femmes girafes, contient des arguments similaires : «Beaucoup d'hypothèses ont été émises par les anthropologues sur la raison du port de ces colliers-spirales. Ce pourrait être pour se protéger contre les morsures de tigres; pour rendre les femmes moins attrayantes aux yeux des autres tribus afin qu'elles ne se marient pas en dehors de la leur ou qu'elles ne soient pas prises en esclavage (...). » http:// fr.wikipedia.org/wiki/Padaung, consulté le 14/05/2012. 
6. Shauna LaTosky, «Reflections on the lip-plates of the Mursi women as a source of stigma and self-esteem ", in Ivo Strecker and Jean Lydall (éds.), The perils of face. Essays on cultural contact, respect and self-esteem in southern Ethiopia, Munster, LIT Verlag, 2006.

7. James Gibson, "The Theory of Affordances", in Robert Shaw and John Bransford (éds.), Perceiving, Acting, and Knowing, John Wiley \& Sons, 1977.

8. Shauna LaTosky, «Reflections on the lip-plates of the Mursi women as a source of stigma and self-esteem », op. cit., p. 373.

9. Ibid., p. 375.

10. Les jeunes filles qui ne portent pas encore de labret utilisent parfois une fine branche de bois souple de 20 à 30 centimètres et placent chaque extrémité d'un côté de la bouche, de sorte que la courbure de la branche tienne lieu de labret.

11. Par exemple, lorsqu'une personne voyage, tous les membres de l'habitation de sa halte sont recouverts d'argile pour les protéger d'éventuels maladies ou mauvais esprits (menenga) qu'elle aurait amené avec lui ; lorsque le danger provient d'un évènement impromptu, comme le passage d'un serpent près d'un enfant, tous les témoins de la scène se peignent le visage ; lorsque on voit dans son rêve un mort ou un évènement tragique, seul le rêveur s'en recouvre.

12. Comme on l'a vu, les saignements tout à fait inopinés suite à une dispute ou une maladresse ne sont pas traités de cette façon. Récurrent s'applique aux conditions et au contexte des saignements davantage qu'au saignement en lui-même.

13. Certaines femmes pour qui la douleur était trop grande ou qui avaient la lèvre trop fragile ne portent pas le labret. Ces femmes se marient et vivent tout à fait comme les autres sans faire l'objet d'un jugement particulier.

14. «Pères » est ici au pluriel car il s'agit d'un terme de référence classificatoire : "père » (shuune, ou dada en adresse affective) fait référence à l'ensemble des membres masculins du clan d'appartenance d'ego, voire à toute la classe d'âge bari (senior elder), qui frappent les teri (senior warrior) lors des rituels initiatiques koma koda (littéralement "genoux frappés ", dans le sens de " genoux à terre »).

15. Il ne faut pas oublier que les femmes ont aussi leur propre duel qui fait suite à la tenue des donga. De lourds bracelets de métal (ula) remplacent les bâtons et les conséquences sont les mêmes, sang qui coule et cicatrices. Les valeurs guerrières et combattantes ne sont ainsi pas le seul privilège des hommes.

16. David Turton, «The same only different: war and dueling as boundary marking rituals in Mursiland, southwestern Ethiopia ", in Tim Cornell (éd.) War and Games, Boydel, and Brewer, Woodbridge, Siffolk, 2002.

17. Il faut noter l'analogie visuelle entre les kichoa volontaires en tiretés et la manière qu'ont les hommes de marquer leurs combats gagnés sur les arbres en pratiquant des lignes d'encoches à l'aide de leur machette qui reprennent ce motif pointillé.

18. LaTosky, dans une note de bas de page, mentionne un labret avec un trou en son milieu. Celui-ci aurait un «sens particulier pour le porteur et la personne qui l'observe » (op. cit., p. 384, je traduis). Il y aurait des tabous de pureté et de pollution associés. On peut y voir, en déployant les conséquences de l'analyse des scarifications proposé ici, que le labret troué reprend le trou de la cicatrice à son compte et qu'il propose une redondance. Explicitant les changements physiologiques de la femme et sous-entendant le sang qui coule, il fait l'objet des mêmes précautions que les évènements qu'il indexe.

19. David Turton, "Lip-plates and "the people who take photographs" ", art. cit., p. 4.

20. La cérémonie juni chibine ( la robe est nouée ») marque ce changement de statut entre la jeune fille (dhole) et l'épouse (moe) et survient bien après le perçage de la lèvre, alors que la femme est déjà mariée. (cf. figure 2) 
21. Michael Houseman, «Note sur quelques propriétés générales de la transformation initiatique », Nouvelle Revue d'ethno-psychiatrie, t. 6, 1986, p. 31-40. Idem, «Quelques configurations de la douleur », in Françoise Héritier (éd.), De la violence II. Paris, Odile Jacob, 1998.

22. J'entends les activités humaines qui laissent une empreinte spécifique permettant d'y voir une volonté consciente de modification et d'agencement. En effet, la plupart des prairies sont modifiées par l'humain au moyen de l'écobuage mais celui-ci n'a pas pour conséquence de former quelque chose d'intentionnellement spécifique ou récurrent.

23. Dans Le Nouveau Monde, de Terrence Malick (The New World), E.-U., 2005, les deux premiers tiers du film se passent aux Amériques. Certaines scènes sont de longs enchaînements de plans à un rythme peu soutenue où la nature est filmée dans son indétermination de forme : champs de blés, branches hautes des arbres à contre-jour, ruisseaux, etc. Comme le propose Jean-Michel Durafour dans « Foulure du regard, plans déboités dans The New World de Terrence Malick 2005 », in Cadrage.net, 2008, http://www.cadrage.net/films/newworld.htm, «la déconstruction perceptive y est d'autant plus remarquable que, dans l'espace diégétique, cette nouvelle expérience esthétique, où les repères habituels sont bouleversés, est également celle des colons qui découvrent le "nouveau monde" ", et de nous inviter dans cette expérience à mieux marquer la rupture avec l'Angleterre : une des premières scènes sur le vieux continent est une succession de plans statiques montrant les perspectives d'un parc où les arbustes et les pierres taillées surprennent par leur régularité et leur alignement, loin de l'aléatoire «naturelle » qui précède. Ce passage brusque de l'un à l'autre, comme l'irruption du labret dans l'environnement en pays Mursi, permet de contraster des formes et les rendre plus étrangères, augmentant par là l'acuité de l'observation et leur saillance.

24. James Gibson, The Ecological Approach of Visual Perception, Lawrence Erlbaum Associates, 1979.

25. Ces moments collectifs cérémoniels constituent la grande majorité des regroupements collectifs en général. L'habitat dispersé et la vie pastorale favorise peu les regroupements. Il n'y a pas de marché et seuls les moments de défrichage des champs sont collectifs et non guidés par des formes spatiales spécifiques.

26. James Gibson, op. cit.

27. Invité par The Pastoralist Communication Initiative à un grand rassemblement de tous les groupes pastoralistes du Sud éthiopien, du Kenya et du Soudan, j'ai été frappé que les Mursi (ce n'étaient pas les seuls) tenaient absolument à compter plusieurs femmes parmi eux, malgré les places rares et les disputes entre hommes pour désigner les représentants. Celles-ci, parfois jeunes et non mariées, n'avaient pas de pouvoir politique et ne prenaient pas part au débat mais il paraissait évident que leur présence durant ces quelques jours était nécessaire. Le fait que pour ces groupes les femmes soient chargées d'ornements et aient des parures très distinctives n'est pas étranger à l'affaire et va au-delà de la marque identitaire de type blason. Les enjeux pragmatiques qui font de la femme un réservoir d'inférence sur les formes sociales est évident, d'autant que les hommes, quant à eux, adoptent dans ces contextes des tenues occidentales sans hésitation.

28. L. Vannutelli et C. Citerni, L'Omo : Viaggi di esplorazione nell'Africa Oreinetale, Milano, U. Hoepli, 1899.

29. Ce conseil de proportions standards est fréquent dans les manuels grands publics de dessin mais différents canons très élaborés se succèdent depuis l'antiquité sur les proportions du corps et du visage, voire notamment la traduction de Les dix livres d'architecture de Vitruve (1673). Pour une référence parmi d'autres sur le système de reconnaissance des visages, voire Vicky Bruce et Andrew Young, «Understanding face recognition », Britain Journal of Psychology, t. 77, 1986, p. 305-327.

30. François Jullien, Cette étrange idée du beau, Chantiers II, Paris, Grasset, 2010.

31. D'après Gérard Genette, dans Jean-Marie Schaeffer, « Objets esthétiques ? », L'Homme, n 170, Espèces d'objets. Paris, EHESS, 2004. 
32. Ibid., p. 42.

33. Melville Herskovits, «The cattle complex in East Africa », American Anthropologist, 1926, 28, p. 230-272, 361-388, 494-528, 633-664.

34. Jean-Baptiste Eczet, «Le peuple des couleurs, entretien avec Pierre Zaoui », Vacarme, $\mathrm{n}^{\circ} 52$, 2010.

35. Idem, "Spreading the self: poetry, color and multilayerd identity among the Mursi ", in Susanne Epple \& Felix Girke (éds.), à paraître.

36. Les Mursi n'ont pas de terme qui recouvre à la fois les humains et le bétail mais les Me'en, avec qui ils partagent le mode de vie, se marient parfois et parlent une langue de même famille, utilisent ce terme (me'en) pour désigner tant les humains que les bovins (Katsuyoshi Fukui, « Coevolution between humans and domesticates: The cultural selection of animal coat-colour diversity among the Bodi ", in Roy Ellen \& Katsuyoshi Fukui (éds.), Redefining nature, Ecology, Culture and Domestication, Berg publishers, 1996).

37. On voit aussi que la condition esthétique qui est de trouver des formes intermédiaires entre humains et bovins disparaît dans les conditions de travestissements induites par les touristes. Là, femmes et enfants, surtout, portent les décorations bovins en défense de phacochère (ngilla) sur la tête comme pour se former des cornes à eux-mêmes. L'un devient l'autre et l'on sort du registre esthétique valorisé pour tomber dans le pastiche, où les Mursi se moquent aussi un peu d'eux-mêmes en jouant au bétail pour faire plaisir aux touristes.

38. Serge Tornay, Les fusils jaunes, Nanterre, Société d'ethnologie, 2001.

39. Idem, « L'énigme des Murle de l'Omo », L'ethnographie, 119² année, n. s.,76-1. 1978.

40. Les Mursi ont aussi conquis un territoire autrefois occupé par les Kwegu. Leslie Woodhead (dans «The Kwegu », Disappearing World, Granada Television, Manchester, UK, 1982), assistée de David Turton, décrit dans un documentaire le rapport de servage qui s'est crée entre eux et les Mursi. Notamment, en contrepartie de la protection par les Mursi et du don de quelques têtes de bétail lors des mariages, les Kwegu servaient de passeurs car eux-seuls maitrisaient la construction de pirogues et daignaient se confronter à l'eau. L'assimilation des Kwegu ne donna toutefois pas lieu à une spécialisation de certaines personnes dans les activités liées au fleuve.

41. Les scarifications Nyangatom sont des lignes droites verticales réalisées en pointillés sur le torse. Le visage est aussi parfois scarifié, et les cicatrices, toujours en pointillés, tournent autour des yeux, formant deux grands ronds, comme des yeux de poissons. Le motif d'écailles et l'agrandissement des yeux participent aussi de ce rapprochement formel qui jamais ne passe le cap de l'inspiration pour devenir de l'imitation.

\section{RÉSUMÉS}

Le plateau labial porté par les femmes Mursi est communément vu comme un objet archaïque voué à disparaître ${ }^{1}$. Au-delà des stéréotypes sur ses raisons d'être, l'argument de cet article est que le labret est une forme synthétisant un ensemble de représentations et d'actions. Il faut donc défaire le feuilleté de sens, les enjeux pragmatiques autant que les effets perceptuels que cette forme simple agence. On y découvre une modification posturale comme intériorisation du regard des hommes; une cicatrice comme initiative engageante dans la condition féminine ; un cercle comme synthèse permanente de formes sociales éphémères; une dé-figuration révélatrice d'un schème esthétique englobant les humains et le bétail. 
The Mursi lip-plate is usually seen as a disappearing sign of backwardness. Beyond a counter argumentation on the stereotypes, I will try to show that the lip-plate has several modes of action, all within the praxis of the body to which it is integral. The layers of meaning, pragmatic challenges and the perceptual impacts accumulated by this simple object must be stripped away. This reveals the following : postural adaptation which internalizes the way that men see women, a scar that represents a binding initiation into the feminine condition, a circular form as a permanent synthesis of ephemeral social formations and a disfigurement that indicates an aesthetic scheme encompassing humans and livestock.

\section{INDEX}

Keywords : adornment, aesthetical pattern, attitude modification, body, lip-plate, scarifications, synthetical form

Mots-clés : labret, modification posturale, scarifications, forme synthétique, modèle esthétique

\section{AUTEUR}

\section{JEAN-BAPTISTE ECZET}

\section{EPHE/Cemaf}

Doctorant à l'Ecole Pratique des Hautes Etudes, Ve section, il mène sa recherche sur l'esthétique des Mursi, un groupe d'agro-pasteurs transhumants du Sud-ouest éthiopien. Aussi bien poétique (noms personnels, poèmes) et rhétorique (discours politiques) que plastique (ornements), l'esthétique mursi est présente au cœur des interactions et des dispositions des acteurs. J.-B. Eczet développe ainsi une approche où les formes politiques sont des formes émergentes de l'esthétique et il revisite les notions de classes d'âge et de cattle complex.

jb_eczet@yahoo.fr 